\title{
Cooperative Job Scheduling and Data Allocation for Busy Data-Intensive Parallel Computing Clusters
}

\author{
Guoxin liu \\ Clemson University \\ Clemson, South Carolina, USA \\ guoxinl@clemson.edu
}

\author{
Haiying Shen, Haoyu Wang \\ University of Virginia \\ Charlottesville, Virginia, USA \\ \{hs6ms,hw8c\}@virginia.edu
}

\begin{abstract}
In data-intensive parallel computing clusters, it is important to provide deadline-guaranteed service to jobs while minimizing resource usage (e.g., network bandwidth and energy). Under the current computing framework (that first allocates data and then schedules jobs), in a busy cluster with many jobs, it is difficult to achieve these objectives simultaneously. We model the problem to simultaneously achieve the objectives using integer programming, and propose a heuristic Cooperative job Scheduling and data Allocation method (CSA). CSA novelly reverses the order of data allocation and job scheduling in the current computing framework, i.e., changing data-first-job-second to job-first-data-second. It enables CSA to proactively consolidate tasks with more common requested data to the same server when conducting deadline-aware scheduling, and also consolidate the tasks to as few servers as possible to maximize energy savings. This facilitates the subsequent data allocation step to allocate a data block to the server that hosts most of this data's requester tasks, thus maximally enhancing data locality and reduce bandwidth consumption. CSA also has a recursive schedule refinement process to adjust the job and data allocation schedules to improve system performance regarding the three objectives and achieve the tradeoff between data locality and energy savings with specified weights. We implemented CSA and a number of previous job schedulers on Apache Hadoop on a real supercomputing cluster. Trace-driven experiments in the simulation and the real cluster show that CSA outperforms other schedulers in supplying deadline-guarantee and resource-efficient services.
\end{abstract}

\section{ACM Reference Format:}

Guoxin liu and Haiying Shen, Haoyu Wang. 2019. Cooperative Job Scheduling and Data Allocation for Busy Data-Intensive Parallel Computing Clusters. In 48th International Conference on Parallel Processing (ICPP 2019), August 5-8, 2019, Kyoto, Japan. ACM, New York, NY, USA, 11 pages. https: //doi.org/10.1145/3337821.3337864

\section{INTRODUCTION}

Data-intensive parallel computing clusters, such as Yarn [4, 35], Spark [5], Cosmos [13] and Mesos [6, 23], have become more important than ever in meeting the needs of big data processing. In

Permission to make digital or hard copies of all or part of this work for personal or classroom use is granted without fee provided that copies are not made or distributed for profit or commercial advantage and that copies bear this notice and the full citation on the first page. Copyrights for components of this work owned by others than ACM must be honored. Abstracting with credit is permitted. To copy otherwise, or republish, to post on servers or to redistribute to lists, requires prior specific permission and/or a fee. Request permissions from permissions@acm.org.

ICPP 2019, August 5-8, 2019, Kyoto, Japan

(C) 2019 Association for Computing Machinery.

ACM ISBN 978-1-4503-6295-5/19/08 . \$15.00

https://doi.org/10.1145/3337821.3337864 the cluster, computing servers also function as storage servers and they form a data storage system (e.g., Hadoop Distributed File System (HDFS) in Yarn). Currently, the clusters employ the computing framework that first allocates data and then schedules jobs (data-first-job-second in short). That is, first, data blocks are randomly distributed to servers $[9,26,31,40]$ and then job schedulers [1, 2, 18, 22, 36, 41-43] allocate job tasks to servers.

However, under the current data-first-job-second computing framework, in a busy cluster with many jobs, it is difficult for a job scheduler to simultaneously achieve deadline guarantee $[17,19,20,22$, 41] (i.e., users' required job deadlines are satisfied), high data locality, and high energy savings simultaneously though they are important for providing high qualify of service (QoS) and reducing cluster capital investment and operation cost. We present the details below.

First, it is difficult to meet the data-locality and deadline guarantee requirements simultaneously in the data-first-job-second computing framework. Job schedulers always try to allocate a task to the server that hosts its requested data (defined as the task's data server) to achieve data locality. With random data allocation, a data server is very likely to host data blocks requested by several submitted tasks, and hence may not provide enough computing slots (i.e., containers) to all the tasks. In this case, some locality-aware schedulers [43] delay scheduling the task, while other locality-aware schedulers $[1,2,18,19,22,24,29,31,41]$ allocate the task to the server that has an available computing slot and is closest to its data server. To meet user deadline requirements, current deadlineaware schedulers $[19,22,41]$ schedule the submitted jobs according to their deadline urgency. Due to a limited number of available computing slots in a busy cluster, it is unlikely to schedule a task in or close to its data server that has an available computing slot in time to satisfy its deadline. Therefore, it is desirable to allocate as many tasks in their data servers as possible to solve the dilemma of achieving high data locality and meanwhile providing deadline guarantee.

Second, it is difficult to meet the data-locality and high-energysaving requirements simultaneously in the data-first-job-second computing framework. A widely used energy saving approach is to put idle servers (that do not execute any job tasks or respond to any data requests) into the sleep mode when other servers can support all workloads, and wake them up when necessary [9, 30, 37, 38]. However, using the data-first-job-second approach, with a high probability, all servers may contain requested data and need to respond to the requests. Even if a server does not store requested data, it may be assigned with a task due to its shortest distance to the task's data server. Fewer sleeping (i.e., inactive) servers increase available computing slots, which improves data locality since 


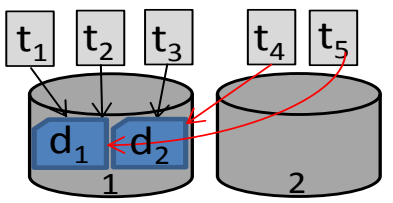

(a) Data-allocation-first

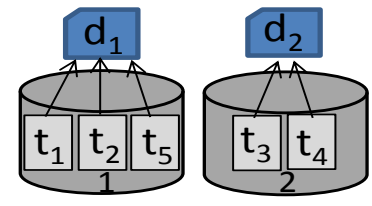

(b) Job-scheduling-first
Figure 1: Data-first-job-second vs. job-first-data-second.

a task has a higher probability to run on its data sever, but increase energy cost. Therefore, it is desirable to solve the dilemma of maximizing the number of sleeping servers (hence energy savings) while achieving data locality.

This paper aims to enhance the performance of simultaneously achieving high data locality, deadline guarantee and high energy savings. We first model the problem to solve the two aforementioned dilemmas using integer programming. Because the problem is NP-hard, we propose a heuristic Cooperative job Scheduling and data Allocation solution (CSA). CSA novelly reverses the order of data allocation and job scheduling in current computing frameworks; that is, it changes data-first-job-second to job-firstdata-second. It enables CSA to proactively consolidate tasks with more common requested data to the same server when conducting deadline-aware scheduling, and also consolidate the tasks to as few servers as possible to maximize energy savings. This facilitates the subsequent data allocation step to allocate a data block to the server that hosts most of this data's requester tasks, thus maximally enhancing data locality.

In the example in Figure 1, each node has three computing slots. Under data-first-job-second, tasks $t_{4}$ and $t_{5}$ must be scheduled to a remote server so they must access their requested data remotely. Under job-first-data-second, tasks $t_{1}, t_{2}$ and $t_{5}$ that request common data block $d_{1}$ are scheduled to server 1 , and tasks $t_{3}$ and $t_{4}$ that request data $d_{2}$ are scheduled to server 2 . Then, allocating data block $d_{1}$ to server 1 and $d_{2}$ to server 2 enable all tasks to access their requested data locally.

Specifically, CSA incorporates three algorithms below.

(1) Requester-consolidation deadline-aware pre-scheduling. It schedules tasks (i.e., data requesters) with common requested data into the same server to facilitate achieving high data locality and consolidates tasks to the fewest servers for energy savings. Also, the pre-scheduling improves the previous deadline-aware schedulers in reducing deadline violations by better ordering the sequence of tasks to be scheduled.

(2) Cooperative data allocation. Based on the determined task schedule, it allocates a data block as close as possible to the server that hosts most of this data's requester tasks in the system in order to maximally achieve data locality, while using the fewest active servers to save energy.

(3) Recursive schedule refinement. It recursively activates sleeping servers if it improves system performance regarding the three objectives and adjusts the task schedule and the data allocation schedule accordingly to further improve the system performance and achieve the tradeoff between data locality and energy savings.

The rest of this paper is organized as follows. Section 2 presents the preliminaries and the problem modeling. Section 3 presents CSA in detail. Section 4 presents the performance evaluation of

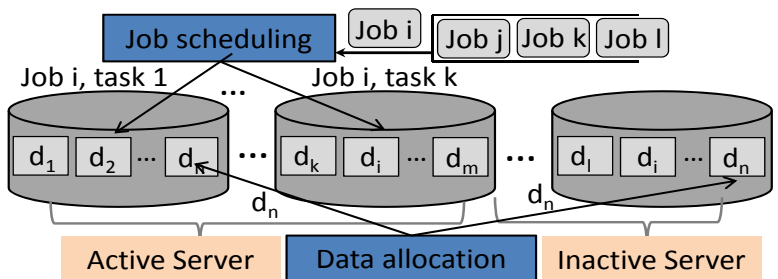

Figure 2: Illustration of job scheduling and data allocation operations.

CSA compared with other methods. Section 5 presents the related work. Section 6 concludes the paper with remarks on our future work.

\section{PROBLEM MODELING}

\subsection{Preliminaries}

In this section, we first present the environment of data-intensive parallel computing clusters. There are many jobs from different users running on a computing cluster. Each user has a set of files, each of which is divided into several blocks (a unit of storage). In order to maintain data availability, each block has $n_{d}$ replicas (e.g., $n_{d}=3$ in HDFS [31]). The process of task and data allocation that assigns tasks and data to servers is shown in Figure 2. We use $D$ to denote the set of data blocks of all users' files, use $d_{i}$ to denote data block $i$ and use $c_{d_{i}}$ to denote its size. We use $S$ to denote the set of all servers, use $s_{k}$ to denote server $k$ and use $C_{s_{k}}^{s}$ to denote the storage capacity of $s_{k}$. As shown in Figure 2, a job is split into several tasks. We use $J$ to denote the set of all tasks of all users' jobs, and use $t_{j}$ to denote task $j$. Server $s_{k}$ 's computing capacity is represented by its total number of computing slots denoted by $C_{s_{k}}^{c}$, which represents the number of tasks that can run simultaneously on $s_{k}$. If block $d_{i}$ requested by a task is missing in its assigned server $s_{k}$, the block needs to be transmitted to $s_{k}$, which introduces network load.

We define task $t_{j}$ 's urgent time as $e_{t_{j}}^{u}=e_{t_{j}}^{d}-\Delta_{t_{j}}$, which is the latest time that $t_{j}$ must start in order not to violate its deadline. Servers that do not serve any tasks or data requests can be put into sleep to save energy as shown in Figure 2. Table 1 lists the main notations used.

\subsection{Problem Formulation}

The problem handled in the paper is: given a group of tasks submitted in each time period $T$, how to schedule the tasks and their associated (i.e., requested) data to simultaneously achieve deadline guarantee, high data locality, and high energy savings? Below, we formulate the deadline-guarantee and resource-efficient scheduling problem in a computing cluster based on integer programming. The problem solution determines job and data allocation schedules to satisfy the deadline requirements of tasks while minimizing the network load (caused by remote data access) and energy cost (determined by the number of active servers) during each time period $T$.

Resource-efficiency objective. The network load of transmitting missing data block $d_{i}$ requested by task $t_{j}$ to its running server $s_{k}$ (denoted by $N_{d_{i}, s_{k}}$ ) is calculated by the method in [11, 28]:

$$
N_{d_{i}, s_{k}}=c_{d_{i}} \cdot l_{d_{i}, s_{k}},
$$


Table 1: Notations.

\begin{tabular}{c|l}
\hline Notation & Description \\
\hline$D / n_{d}$ & the set of all blocks / the number of replicas of a block \\
\hline$D_{t_{j}}$ & the set of data blocks requested by task $t_{j}$ \\
\hline$S / s_{k}$ & the set of servers / server $k$ \\
\hline$J / t_{j}$ & the set of all tasks of all jobs / task $j$ \\
\hline$D_{s_{k}}$ & the set of data blocks stored in server $s_{k}$ \\
\hline$d_{i} / c_{d_{j}}$ & data block $i\left(d_{i} \in D\right) /$ size of data block $d_{i}$ \\
\hline$S^{a} / S^{i n}$ & the set of active servers / the set of inactive servers \\
\hline$S_{t_{j}}^{a}$ & the set of active data servers that serve task $t_{j}$ \\
\hline$C_{s_{k}}^{s} / C_{s_{k}}^{c}$ & server $s_{k}$ 's storage capacity /server $s_{k}$ 's computing capacity \\
\hline$J^{v}$ & the set of tasks whose deadlines are violated \\
\hline$x_{d_{i}, s_{k}}$ & whether a replica of $d_{i}$ exists in $s_{k}$ \\
\hline$y_{t_{j}}, s_{k}$ & whether task $t_{j}$ is assigned to run on $s_{k}$ \\
\hline$z_{d_{i}, t_{j}}$ & whether data block $d_{i}$ is requested by task $t_{j}$ \\
\hline$T$ & a time period during which tasks in $J$ are scheduled \\
\hline$e_{i}$ & the end time point of $i^{t h}$ unit time interval in $T$ \\
\hline$e_{t_{j}}^{s} / \Delta_{t_{j}} / e_{t_{j}}^{u}$ & task $t_{j}$ 's submission time / execution time period / urgent time \\
\hline$e_{t_{j}}^{d} / e_{t_{j}}^{r}$ & task $t_{j}$ 's deadline / actual start time \\
\hline$N_{d_{i}, s_{k}}$ & the smallest network load to transmit data $d_{i}$ to server $s_{k}$ \\
\hline
\end{tabular}

where $l_{d_{i}, s_{k}}$ denotes the shortest path length (measured by the number of hops) for transmitting a replica of block $d_{i}$ to server $s_{k}$ among several active servers storing $d_{i}$ in the system. We use $x_{d_{i}, s_{k}}$ to denote whether block $d_{i}$ is stored in server $s_{k}$. Then, the set of blocks stored in server $s_{k}$ can be represented by $D_{s_{k}}=$ $\left\{d_{i} \mid x_{d_{i}, s_{k}}=1 \wedge d_{i} \in D\right\}$. Thus, we can calculate the total network load for all tasks that will execute during $T$ :

$$
N=\sum_{t_{j} \in J} \sum_{s_{k} \in S} \sum_{d_{i} \in\left(D_{t_{j}} \backslash D_{s_{k}}\right)} N_{d_{i}, s_{k}} \cdot y_{t_{j}, s_{k}},
$$

where $D_{t_{j}}$ denotes the set of data blocks requested by task $t_{j}, D_{t_{j}} \backslash$ $D_{s_{k}}$ denotes task $t_{j}$ 's requested data blocks that are not stored in server $s_{k}$ and need remote access, and $y_{t_{j}, s_{k}}$ denotes whether task $t_{j}$ is assigned to run on $s_{k}$. Resource-efficient job scheduling and data allocation methods need to minimize $N$ by improving data locality.

We use $S^{a}$ and $S^{i n}$ to denote the set of all active and inactive servers during $T$, respectively. $S^{a}$ consists of the servers that serve the tasks in $J$ (denoted by $S_{t}^{a}$ ) and the servers that serve data requests (denoted by $S_{d}^{a}$ ).

$S_{t}^{a}=\left\{s_{k} \mid s_{k} \in S \wedge \sum_{t_{j} \in J} y_{t_{j}, s_{k}}>0\right\}$ and

$S_{d}^{a}=\left\{s_{i} \mid s_{i}=s_{d_{i}, s_{k}} \wedge d_{i} \in D_{t_{j}} \backslash D_{s_{k}} \wedge y_{t_{j}, s_{k}}=1\right\}$,

where $s_{d_{i}, s_{k}}$ denotes the active server storing $d_{i}$ that is closest to $s_{k}$. Then, in order to save energy, we need to minimize:

$$
\left|S^{a}\right|=\left|S_{t}^{a} \cup S_{d}^{a}\right| \text {. }
$$

Deadline-guarantee objective. To provide deadline guarantee service to task $t_{j}$, we need to satisfy $e_{t_{j}}^{s} \leq e_{t_{j}}^{r} \wedge e_{t_{j}}^{r}+\Delta_{t_{j}} \leq e_{t_{j}}^{d}$, where $e_{t_{j}}^{r}$ is $t_{j}$ 's actual running start time. We use $J^{v}$ to denote the set of tasks whose deadlines are violated:

$$
\left|J^{v}\right|=|J|-\left|\left\{t_{j} \mid t_{j} \in J \wedge e_{t_{j}}^{s} \leq e_{t_{j}}^{r} \wedge e_{t_{j}}^{r}+\Delta_{t_{j}} \leq e_{t_{j}}^{d}\right\}\right| .
$$

A scheduler needs to minimize $\left|J^{v}\right|$. Note that we aim to ensure that each job completes before its deadline rather than minimizing the job completion time.

Constraints. The following constraints are based on the default protocols in the current Yarn $[4,35]$. We need to create a valid data allocation schedule that meets the data availability requirement and the storage capacity constraint. That is, each data block needs to have $n_{d}$ replicas:

$$
\forall d_{i} \in D, \sum_{s_{k} \in S} x_{d_{i}, s_{k}}=n_{d}
$$

and

$$
\forall s_{k} \in S, \sum_{d_{i} \in D_{s_{k}}} c_{d_{i}} \leq C_{s_{k}}^{s} ;
$$

A valid job schedule needs to ensure that at any time during $T$, there are no more than $C_{s_{k}}^{c}$ tasks running in any server $s_{k} \in S$ :

$$
\forall s_{k} \in S, n_{s_{k}} \leq C_{s_{k}}^{c},
$$

where $n_{s_{k}}$ denotes the number of concurrently running tasks on $s_{k}$ at a time in $T$.

Problem statement. We combine Objectives expressed by Equations (2), (3) and (4) together to a single objective to model the deadline-guarantee and resource-efficient schedule problem. Assume that the cluster operator considers that energy saving (Equation (3))

$$
\begin{aligned}
& \min \left(\left(1+\beta^{2}\right) \cdot \frac{N \cdot\left|S^{a}\right|}{\left(\beta^{2} \cdot N+\left|S^{a}\right|\right)} /\left(|J|-\left|J^{v}\right|\right)\right) \\
& \text { subject to : Constraints (5), (6) and (7) }
\end{aligned}
$$

is $\beta$ times as importance as network load minimization (Equation (2)). Then, based on [34] that provides a F-score (or $\left.F_{\beta}\right)$ formula to represent such relationship in combining two metrics, we can model the problem as an integer program: Formula (8) first combines Objectives (2) and (3) so that it minimizes the network load and energy cost with the consideration of weight $\beta$ [34]. Then, Formula (8) combines Objective (4) of maximizing the number of tasks without deadline violations.

A simple reduction from this problem is equivalent to the generalized assignment problem in [21] and can be used to prove that the problem is NP-hard. We skip formal proof due to space limit. Then, we introduce a heuristic solution to generate the job schedule and data allocation schedule in Section 3.

\section{COOPERATIVE JOB SCHEDULING AND DATA ALLOCATION}

To solve the problem above, we propose a heuristic Cooperative job Scheduling and data Allocation method (CSA). The tasks that can be predicted or reported from the users are called planned tasks; otherwise, non-planned tasks. As in [25], CSA runs periodically after each time period $T$, and uses offline pre-scheduling that calculates the schedule for all planned tasks that are planned to submit in the next time period $T$. In $T$, the actually submitted planned tasks and their associated data are scheduled based on the determined job schedule and data allocation schedule from CSA, and the submitted non-planned tasks are scheduled to meet their deadlines without interrupting the determined schedules. As the planned jobs can finish earlier because of high data locality and lower network consumption from CSA, it leaves more computing resources and network bandwidth to non-planned jobs. Thus, the performance of both planned and non-planned jobs, and the entire system can be improved by CSA. CSA is mainly designed for the jobs with associated data that needs to be stored into the system rather than already existing in the system though it can be directly used. CSA incorporates the following algorithms: 
(1) Requester-consolidation deadline-aware pre-scheduling (Section 3.1). It calculates job schedule that specifies the time and the server to schedule each planned task that will be submitted in the next $T$. It proactively consolidates tasks with more common requested blocks to the same server as shown in Figure 1(b); $t_{1}, t_{2}$ and $t_{5}$ requesting $d_{1}$ are pre-scheduled to server 1 . It also improves the deadline guarantee performance of existing deadline-aware schedulers by better ordering the sequence of tasks to be scheduled.

(2) Cooperative data allocation (Section 3.2). Facilitated by the above algorithm, this algorithm allocates data to be locally shared by as many tasks as possible (e.g., $d_{1}$ is allocated to server 1 in Figure $1(\mathrm{~b})$ ), thus avoiding the situation that a task cannot be allocated to its data server due to computing slot limit and maximally achieving data locality in the system.

(3) Recursive schedule refinement (Section 3.3). This algorithm is optional. Before actual data allocation, it can be used to recursively refine the job schedule and data allocation schedule (after activating some sleeping servers) to further improve the system performance regarding the three objectives and achieve the tradeoff between data locality and energy savings with specified weights.

\subsection{Requester-Consolidation Deadline-Aware Pre-Scheduling}

This algorithm incorporates two parts: i) common-data requester consolidation, and ii) deadline-aware first-in-first-out (FIFO) prescheduling. Part i) schedules each planned task in order to consolidate the tasks requesting more common requested blocks to the same server. Part ii) determines the order of tasks to be scheduled to reduce the deadline violations and more fully utilize computing slots in the system over the existing deadline-aware schedulers.

3.1.1 Common-data Requester Consolidation. In practice, many jobs may process the same dataset. For example, a user may submit different jobs along with the same dataset such as spam detection jobs and advertisement recommendation jobs for the same dataset from an online social network. Amazon provides Google books ngram dataset with its name and path in Amazon's Hadoop storage system, so different users can submit different jobs on this dataset such as statistic jobs for user different activities. By taking advantage of this fact, in order to improve data locality, CSA novelly aims to allocate tasks that request as many of the same data blocks as possible to the same server. This facilitates the subsequent data allocation algorithm to store a data block requested by many tasks on a server to this server. Such cooperative task scheduling and data allocation improve data locality and hence reduce network load.

In a cluster, the job scheduling is conducted in a real-time manner, that is, a job is scheduled once it is submitted if a computing slot is available. Therefore, for the tasks that will be submitted in the next $T$, we conduct offline-scheduling after each small time interval, i.e., schedule submitted tasks by each time point $e_{i}$ in $T$ to the computing slots available at $e_{i}$. When CSA conducts the offlinescheduling for future time $e_{i}$, we call the tasks that will be submitted before $e_{i}$ submitted tasks.

To achieve the goal of task consolidation, when assigning task $t_{j}$ to a server among all active servers with an available computing slot at $e_{i}$ in $T, C S A$ allocates $t_{j}$ to the server, which requires the smallest size of data to be transmitted for $t_{j}$ 's running. Specifically,

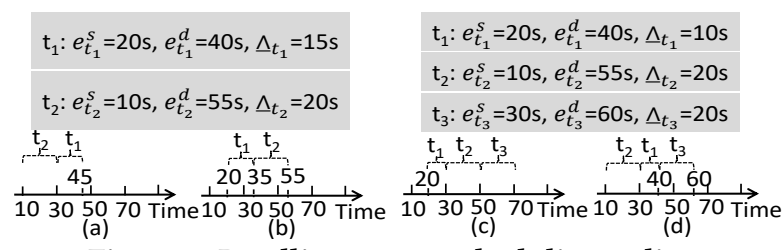

Figure 3: Deadline-aware scheduling policy.

for each active server $s_{k}$, CSA maintains the information of all data blocks $\left(D_{s_{k}}\right)$ requested by the tasks that have been scheduled to each server $s_{k}$. For each server candidate, CSA calculates the total size of missing blocks requested by $t_{j}: C_{t_{j}, s_{k}}=\sum_{d_{i} \in D_{t_{j}} \backslash D_{s_{k}}} c_{d_{i}}$. The server with the smallest $C_{t_{j}}, s_{k}$ is selected to execute $t_{j}$. If there are more than one server with equal $C_{t_{j}, s_{k}}, t_{j}$ is scheduled to a randomly selected server among them. As more and more tasks are scheduled to servers, CSA proactively consolidates tasks with more common requested data to the same server as shown in Figure 1(b).

3.1.2 Deadline-aware FIFO Pre-scheduling. This part handles the problem about how to order the tasks to be scheduled. We improve the existing deadline-aware schedulers by deadline-aware pre-scheduling that schedules the jobs planned to submit in the next $T$ and better ordering the tasks for scheduling. Below, we first show the importance of pre-scheduling for deadline guarantee. Then, we present a problem that may cause deadline violation in pre-scheduling and propose a method to order the tasks to be scheduled to avoid this problem.

We define tight-deadline and loose-deadline tasks as the tasks with deadlines close to and far away from the scheduling time $e_{i}$, respectively. Figures 3 (a) and (b) show one scenario indicating the current schedulers' drawback. That is, only scheduling submitted tasks [1, 2, 18, 19, 22, 31, 41, 43] may violate the deadlines of tightdeadline tasks that will be submitted in the future if all computing slots are occupied. As shown in Figure 3(a), there is one server with one computing slot available from $10 \mathrm{~s}$ to $50 \mathrm{~s}$. Since task $t_{2}$ is submitted before $t_{1}$, current schedulers schedule $t_{2}$ to the server first and then $t_{1}$. Then, $t_{1}$ is scheduled at $e_{t_{2}}^{s}+\Delta_{t_{2}}=30 \mathrm{~s}$, which is later than its urgent time $e_{t_{1}}^{u}=25 \mathrm{~s}$ and will complete at $45 \mathrm{~s}$. As a result, $t_{1}$ 's deadline is violated. Therefore, a job scheduler should consider not only current submitted tasks but also the planned tasks that will be submitted later. Using pre-scheduling, as shown in Figure 3(b), being aware of the deadlines of both $t_{1}$ and $t_{2}$, a pre-scheduler schedules $t_{2}$ after $t_{1}$ to meet their deadlines.

However, pre-scheduling brings about a problem. If we use Earliest Urgent Time First (EUT) [19, 22, 41] in pre-scheduling, a computing slot may be idle since it is assigned to a future task while a submitted task must wait, which leads to computing resource waste and may cause deadline violations. For example, in Figures 3(c) and (d), there is one server with one computing slot and three tasks, and their urgent times are $e_{t_{1}}^{u}=30 s, e_{t_{2}}^{u}=35 \mathrm{~s}$, and $e_{t_{3}}^{u}=40 \mathrm{~s}$ and thus follow $e_{t_{1}}^{u}<e_{t_{2}}^{u}<e_{t_{3}}^{u}$. Figure 3(c) shows a schedule sequence using EUT, which is $t_{1}, t_{2}$ and $t_{3}$. However, the computing slot during $10 \mathrm{~s}-20 \mathrm{~s}$ is idle and $t_{3}$ is scheduled at $e_{t_{1}}^{s}+\Delta_{t_{1}}+\Delta_{t_{2}}=50 \mathrm{~s}$, which is later than its urgent time $e_{t_{3}}^{u}=40 \mathrm{~s}$. Then, task $t_{3}$ 's deadline is violated. If $t_{2}$ submitted earlier than $t_{1}$ does not need to yield to $t_{1}$ as shown in Figure $3(\mathrm{~d}), t_{3}$ is scheduled at $e_{t_{2}}^{s}+\Delta_{t_{1}}+\Delta_{t_{2}}=40 \mathrm{~s}$, 
the computing slots are fully utilized and all tasks' deadlines are guaranteed.

Therefore, when task $t_{k}$ with an earlier urgent time and a later submission time competes a computing slot with task $t_{j}$ with an earlier submitted task and a later urgent time $\left(\left(e_{t_{k}}^{u}<e_{t_{j}}^{u}\right) \wedge\left(e_{t_{j}}^{s}<\right.\right.$ $\left.e_{t_{k}}^{s}\right)$ ), then if scheduling $t_{j}$ first can still provide deadline guarantee for $t_{k}, t_{k}$ should not be scheduled earlier. That is, when $t_{k}$ should be scheduled first using EUT while $t_{j}$ should be scheduled first using FIFO, then $t_{j}$ is scheduled first to the computing slot only if it still provides deadline guarantee for $t_{k}$. Hence, we hope to concurrently consider FIFO when determining the order of tasks to be scheduled. Accordingly, we propose deadline-aware FIFO prescheduling policy that combines EUT with FIFO scheduling. In this policy, for a task $t_{j}$, it should be scheduled after the tasks with urgent times earlier than $t_{j}$ 's urgent time (based on EUT) except the tasks that are submitted after $t_{j}$ and have urgent times after $t_{j}$ 's completion (based on FIFO). We call the set of tasks that should be scheduled before $t_{j}$ its predecessor list and denote it at scheduling time $e_{i}$ in the next $T$ by $J_{e_{i}, t_{j}}$. It is derived from the list of all tasks submitted by $e_{i}$ in the next $T$ and will be submitted in the remaining time in the next $T$ that have not been scheduled (denoted by $\left.J^{u}\right)$ :

$$
J_{e_{i}, t_{j}}=\left\{t_{k} \mid\left(t_{k} \in J^{u}\right) \wedge\left(e_{t_{k}}^{u}<e_{t_{j}}^{u}\right) \wedge \neg\left[\left(e_{t_{j}}+\Delta_{t_{j}} \leq e_{t_{k}}^{u}\right) \wedge\left(e_{t_{j}}^{s}<e_{t_{k}}^{s}\right)\right]\right\} .
$$

We use $n_{c}$ to denote the number of available computing slots in the system at $e_{i}$. If $n_{c} \geq\left|J_{e_{i}, t_{j}}\right|+1$, it means $t_{j}$ 's scheduling will not delay the scheduling of the tasks in its predecessor list, and it can be scheduled to an available computing slot. Assume task $t_{j}$ is scheduled to server $s_{k}$. The computing slot in $s_{k}$ may be available at a time before $e_{i}$, denoted by $e_{e_{i}, s_{k}}^{c}$. Then, $t_{j}$ 's start time should be the later time among $t_{j}$ 's submission time and $e_{e_{i}, s_{k}}^{c}$, i.e., $\operatorname{Max}\left\{e_{e_{i}, s_{k}}^{c}, e_{t_{j}}^{s}\right\}$. This way, $t_{j}$ can start as early as possible in order to complete earlier and meanwhile fully utilize computing resource. To achieve energy-efficiency, all servers are deactivated initially, and an inactive server is activated only when there are no available computing slots and there is a task that has not been scheduled by its urgent time.

3.1.3 Algorithm for the Requester-Consolidation Deadline-Aware Pre-Scheduling. Algorithm 1 shows the procedure of our pre-scheduling algorithm combining the above two parts. With an input of a group of planned tasks in the next $T$ and servers $<J^{u}, S>$, the algorithm generates a job schedule that specifies the time and the server to allocate each task. We use $J_{e_{i}}^{w}$ to denote the task waiting list, which includes all submitted tasks that have not been scheduled by sched-

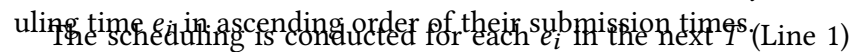
and then for each task in $J_{e_{i}}^{w}$ (Line 2). This way, we can consider both EUT and FIFO in our Deadline-aware FIFO Pre-scheduling. That is, for Objective (4), at $e_{i}$, for each $t_{j} \in J_{e_{i}}^{w}$, if its urgent time $e_{t_{j}}^{u} \leq e_{i}$, it must be scheduled, otherwise, if scheduling it won't violate the deadlines of its predecessors (i.e., there are enough computing slots for its predecessors, $\left.n_{c} \geq\left|J_{e_{i}, t_{j}}\right|+1\right)$, it is scheduled. In the former case, when there is no available computing slot, a sleeping server is activated (Lines 4-7) according to the energy saving policy to achieve Objective (3). To schedule $t_{j}$ among multiple servers, a server is chosen using the Common-data Requester

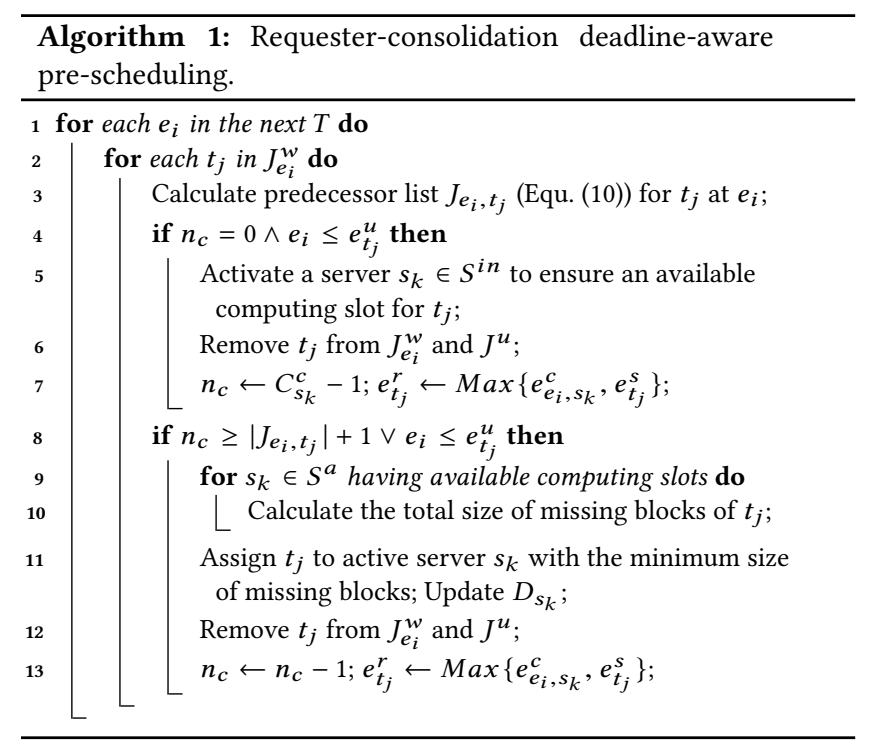

Consolidation method (Lines 9-13), which generates the minimum bandwidth consumption for transferring missing blocks requested by $t_{j}$. Finally, corresponding parameters are updated and the start time of $t_{j}$ is decided (Lines 6-7, 12-13).

3.1.4 Scheduling Non-planned Jobs. Since in Algorithm 1, the computing resources during $T$ are reserved for planned jobs, the tasks of non-planned jobs should be scheduled without taking up a reserved computing slot. Thus, in online scheduling, CSA can assign non-planned task $t_{j}$ to a server having an available computing slot lasting $\Delta_{t_{j}}$ under the calculated job scheduler. The constraint of lasting $\Delta_{t_{j}}$ is used to preserve the schedule in the pre-scheduling. Task $t_{j}$ is scheduled to the qualified server with the minimum size of missing blocks of $t_{j}$.

\subsection{Cooperative Data Allocation}

After job pre-scheduling, CSA then conducts data allocation (for the data associated with the tasks in the next $T$ denoted by $D_{w}$ ) among servers with the objective of network load minimization under Constraints (5) and (6). We use $z_{d_{i}, t_{j}}$ to denote whether block $d_{i}$ is requested by task $t_{j}$ during $T$. The network load of requesting $d_{i}$ (denoted by $N_{d_{i}}$ ) is calculated by $N_{d_{i}}=\sum_{t_{j} \in J} \sum_{s_{k} \in S} y_{t_{j}, s_{k}}$. $z_{d_{i}, t_{j}} \cdot N_{d_{i}, s_{k}}$, which is determined by the request frequency, the data size and the number of transmission hops [11,28]. For each data block $d_{i}, C S A$ allocates $d_{i}$ to the server which generates the minimum $N_{d_{i}}$. One question here is how to set the order of data blocks to be scheduled. We define the transmission size of $d_{i}$ as the product of its requested frequency and block size, $\sum_{t_{j} \in J} z_{d_{i}, t_{j}} \cdot c_{d_{i}}$. Since data blocks with a larger transmission size tend to generate higher network load, giving them higher priority to be scheduled can reduce the total network load more. Thus, we sort all data blocks in a decreasing order of the transmission size for data block allocation. In order to maintain the effectiveness of energy savings achieved by the pre-scheduling algorithm, only when there are no active servers that have enough storage capacity, $d_{i}$ is allocated to an inactive server. The inactive server is activated if it stores the first replica of $d_{i}$ to ensure data availability. 

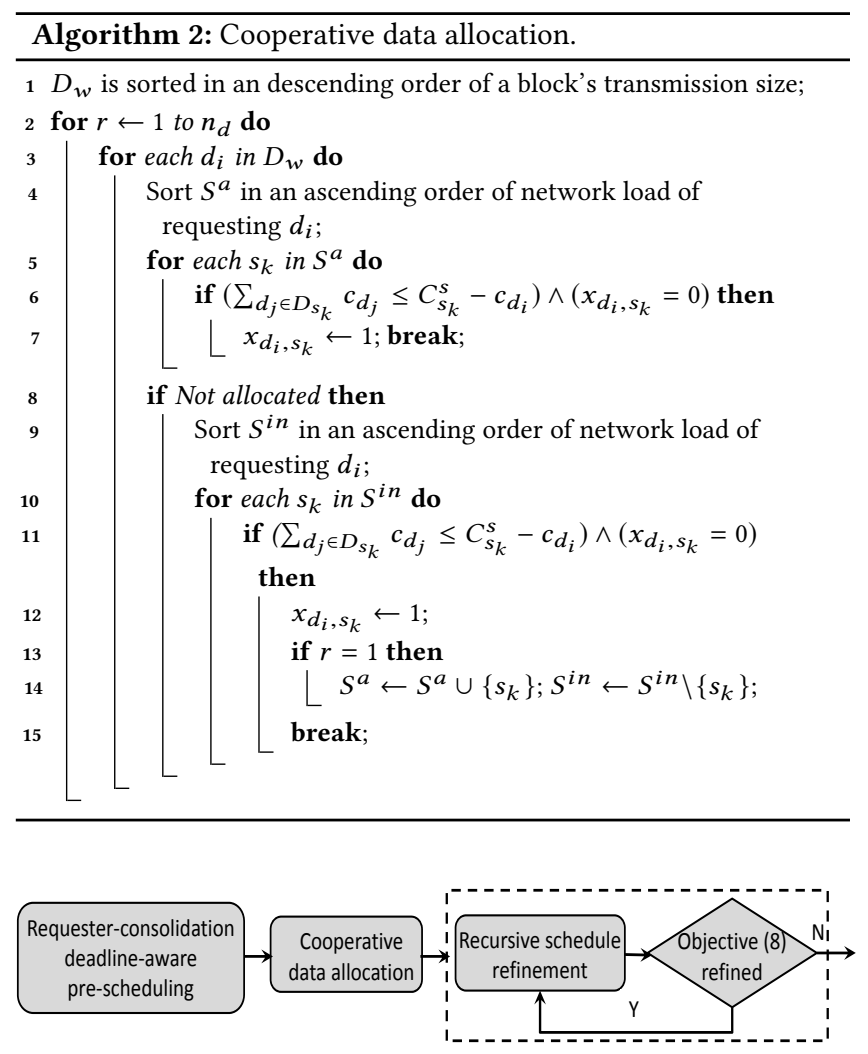

Figure 4: Recursive schedule refinement process.

Algorithm 2 shows the pseudocode of the cooperative data allocation algorithm. We sort all blocks in $D_{w}$ in a descending order of their transmission sizes, generate $n_{d}$ replicas for each data block to meet Constraint (5), and allocate the blocks to servers in the topdown manner (Lines 1-3). For each data block $d_{i}$, we choose the active server $s_{k}$ that generates the lowest network load of $d_{i}$ (Lines 4-7) to assign $d_{i}$ (which improves data locality) while still satisfying Constraint (6). If all active servers without $d_{i}$ 's replica do not have enough storage capacity to store $d_{i}$ (Line 8), for each inactive server $s_{k} \in S^{i n}$, we calculate the network load of $d_{i}$ by assuming that $d_{i}$ is stored in active $s_{k}$. Then, we choose the inactive server that generates the lowest network load to store it (Lines 9-12). In order to make sure that all data requests will be responded, the servers containing the first replica of $b_{i}$ cannot be put into sleep. That is, if a first data replica is assigned to a server in $S^{i n}$, this server is activated and joins $S^{a}$ (Lines 13-14). Therefore, there exits at least one replica of any block to respond to data requests in the system.

\subsection{Recursive Schedule Refinement}

The pre-scheduling and data allocation algorithms maximize the energy savings and enhance data locality. According to Algorithm 2, some replicas of data blocks may be stored in inactive servers in order to maintain the energy savings achieved by Algorithm 1. Activating some inactive servers may improve data locality and reduce network load due to fewer transmission hops for requesting data blocks. In addition, cluster operators may wish to give different weights to data locality and energy savings in considering resource usage reduction. Therefore, we further propose the recursive schedule refinement algorithm, which is optional to be used in CSA.

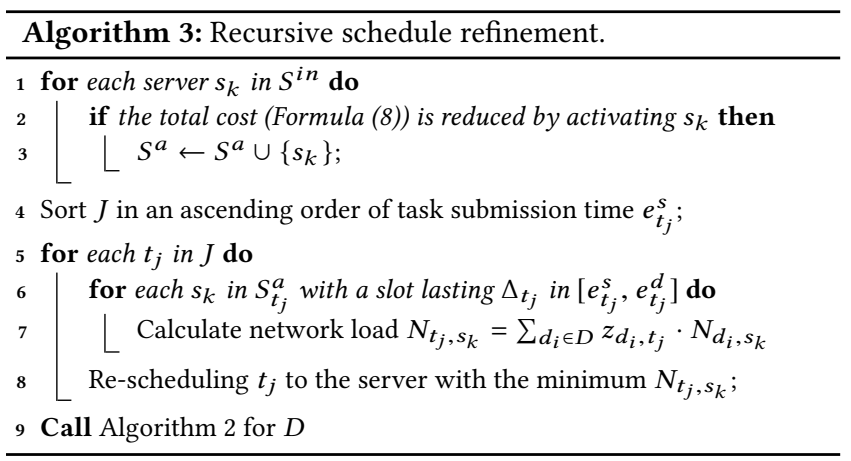

As shown in Figure 4, this algorithm is executed after the execution of the cooperative data allocation algorithm (that determines the data allocation schedule) and before the actual data allocation. It includes a task schedule refinement algorithm and the cooperative data allocation algorithm introduced in Section 3.2. Algorithm 3 shows the pseudocode of this recursive schedule refinement algorithm. For each server $s_{k}$ in the inactive set $S^{i n}$ (Line 1), after activating it, the data allocation and task schedules keep the same but the energy cost $\left(\left|S^{a}\right|\right)$ is increased. Due to the data locality awareness, the new activated server needs to respond data requests to reduce the network load $(N)$ if it leads to a smaller $l_{d_{i}, s_{k}}$ in Equation (1). We measure the total cost based on Formula (8) before and after we activate $s_{k}$, respectively. If the cost is reduced, this server is put into the active set (Lines 2-3).

After the above server activation, since the set of active servers is changed, re-scheduling tasks may lead to lower network load. In the re-scheduling, we sort all tasks in $J$ in an ascending order of their submission time $e_{t_{j}}^{s}$ (Line 4 ). For each $t_{j}$ in $J$, we calculate the network load for fetching missing data blocks requested by $t_{j}$ if it is re-scheduled to each of its data server that has a computing slot lasting $\Delta_{t_{j}}$ between $t_{j}$ 's submission time and its deadline (i.e., without deadline violation) calculated by $\sum_{d_{i} \in D} z_{d_{i}, t_{j}} \cdot N_{d_{i}, s_{k}}$ (Lines 5-7). Then, we re-schedule $t_{j}$ to its data server that generates the lowest network load (Line 8). This step ensures that Constraint (7) is met, the energy savings and the number of tasks without deadline violations will not be changed while data-locality can be improved, so that the total cost for Objective (8) can be reduced. As a result, a valid task schedule with a lower cost for Objective (8) is generated. After the task schedule refinement, the task schedule has been changed. Next, the algorithm conducts the cooperative data allocation algorithm again under the new task schedule for $D$ (Line 9). We call one time execution of the algorithm an iteration in the recursive schedule refinement process. Each iteration improves the data locality, but increases the energy cost while maintaining the same number of tasks without deadline violations. In order to minimize the cost in Objective (8), CSA recursively refines the task schedule and data allocation schedule as shown in Figure 4 and achieves a tradeoff between energy savings and data locality with specified weights $(\beta)$. This process terminates when the total cost 


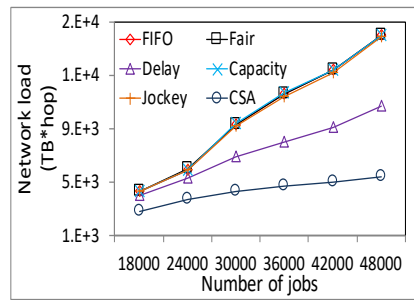

(a) Simulation

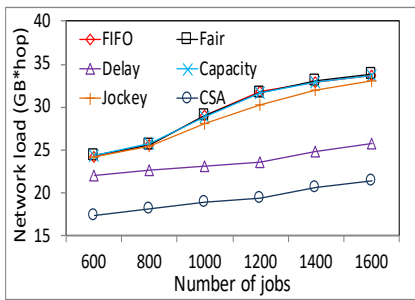

(b) Real cluster

Figure 5: Network load.

after an iteration has no or little cost improvement over the last iteration.

\section{PERFORMANCE EVALUATION}

We conducted trace-driven experiments on both simulation and a real Hadoop cluster to evaluate CSA's performance in supplying deadline-guaranteed and resource-efficient service in comparison with other methods.

Simulated computing cluster. In the simulation, there were 3000 servers in a computing cluster as in the Facebook's Hadoop cluster trace [15]. The topology of this computing cluster is a typical fat-tree topology [8] with three layers. The storage capacity of each server was randomly chosen from $\{6 \mathrm{~TB}, 12 \mathrm{~TB}, 24 \mathrm{~TB}\}$ according to the Facebook cluster [3, 40]. Without specific declaration, the computing capacity of each server was set to 8 computing slots by default according to [10]. The default block size was set to $64 \mathrm{MB}$, and the number of replicas of a block was set to 3 [31]. We set the total size of data to $10 \mathrm{~PB}$ in order to fit the data to the 3000 node cluster, since there is tens of PBs of data in the Facebook's cluster [33].

Simulated workloads. We set the time period $T$ to 24 hours as in [25]. Unless otherwise specified, we simulated 100 users and generated 24000 MapRedue jobs to submit in $T$, which were randomly chosen from all jobs in the Facebook's Hadoop cluster trace in [15]. The jobs' submission time distribution follows the same distribution in the trace [15]. The sizes of the requested data and the output results of a job were set based on the trace [15]. Each requested block of a job was randomly chosen from all data blocks. Each task was assigned with $256 \mathrm{MB}$ requested data (i.e., 4 data blocks) since each map task handles the same amount of data in Hadoop [31]. We measured the execution time of each task, $\Delta_{t_{j}}$, using offline running as in $[19,22]$ on the Palmetto supercomputing cluster [7] with 7718 -core nodes. We set the deadline of each task as $e_{t_{j}}^{d}=e_{t_{j}}^{s}+\alpha \cdot \Delta_{t_{j}}$, where $\alpha$ follows a Bounded Pareto distribution with a shape as 2 and a lower bound and an upper bound as $105 \%$ and $150 \%$, respectively. By default, $\beta$ in Objective (8) equals 8 , which means that the cluster operator considers energy saving 8 times as important as network load saving.

Real cluster. We implemented CSA and compared previous job schedulers on Apache Hadoop on the Palmetto supercomputing cluster [7]. Due to the usage limitation, we randomly chose 100 nodes to form a computing cluster with a fat-tree topology [8]. Since the scale of the cluster is $1 / 30$ of the cluster in the simulation, we reduce the default total number of jobs to $1 / 30$, i.e., 815 randomly selected jobs. Due to the local storage limitation, we reduce the storage capacity of a server and the sizes of the requested

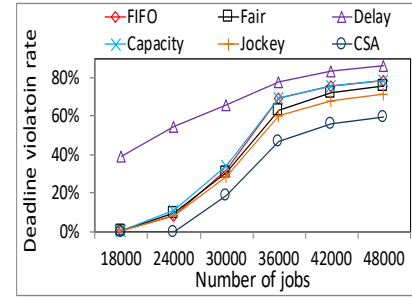

(a) Simulation

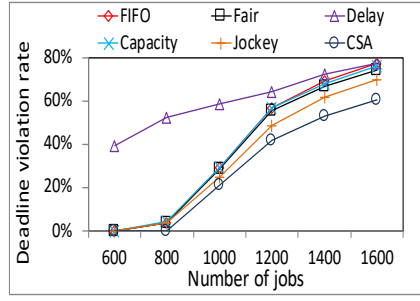

(b) Real cluster
Figure 6: Deadline violation rate.

data and output of each task to $1 / 1000$ of their original sizes. All other settings keep the same as in the simulation.

Compared methods. We compared CSA with the following job schedulers: Delay [43], FIFO [31], Fair [2], Capacity [1] and fockey [19]. These schedulers first randomly allocate data and then allocate tasks to nodes [31]. Delay delays the scheduling of a task until one of its data servers has a computing slot. The other schedulers assign a task to the server that is the closest to its requested data, and they use different criteria in determining which task in the submitted tasks should be scheduled first. FIFO schedules a task with the earliest submission time fist. Fair aims to achieve fair sharing of resources among all jobs, that is, the jobs have the same average number of computing slots over time. Capacity aims to satisfy users' initial demands of computing capacity, (i.e., the number of computing slots). In our test, we assume equal computing capacity demands among users. fockey schedules the task with the earliest deadline first. In our test, when scheduling a task $t_{j}$, the above schedulers choose one of its data servers that has the largest number of data blocks requested by $t_{j}$ or the server closest to this data server if it does not have a computing slot. We run each experiment for 10 times and report the average value.

\subsection{Comparison of Different Schedulers}

In this section, we measured the performance of all schedulers in a busy cluster with large data processing workloads. We varied the number of jobs from 18000 to 48000 with a step size of 6000 in the simulation, and varied it from 600 to 1600 with a step size of 200 in the real cluster experiment. When the number of jobs is larger than 24000 in simulation and 800 in the real cluster, the workload exceeds the computing capability of the cluster.

Figures 5(a) and 5(b) show the network load of each scheduler in simulation and the real cluster, respectively. They show that the network load follows fockey $\approx F I F O \approx$ Capacity $\approx$ Fair $>$ Delay $>$ CSA. All schedulers except Delay and CSA assign tasks to the servers that are the closest to their requested data. Therefore, they have similar performance. Delay always schedules a task to its data server having the largest number of its requested data blocks, which achieves better data locality than the previous schedulers. However, due to the random data-allocation-first, a task's requested data blocks may not in the same server, which generates certain network load. CSA has a better performance than Delay by its novel job-first-datasecond computing framework, which helps to allocate as many a task's requested data to the task's assigned server as possible. We also see that with the growth of the number of jobs, the network load of CSA increases the slowest, and that of Delay increase slower than other schedulers. The result implies that CSA can constrain 


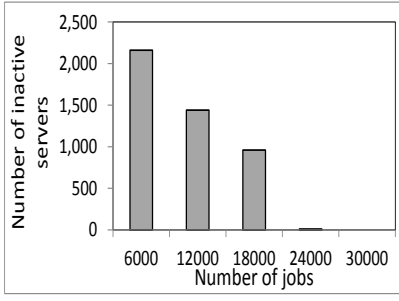

(a) Simulation

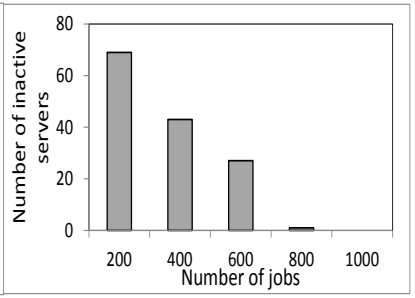

(b) Real cluster
Figure 7: Energy savings.

the network load even in a very busy cluster. The results indicate that CSA achieves much better data locality than all other schedulers and the effectiveness of the job-first-data-second computing framework.

We measured the deadline violation rate, which is the percentage of tasks whose deadlines are violated. Figures 6(a) and 6(b) show the deadline violation rate of all schedulers versus the number of jobs in simulation and the real cluster, respectively. The result follows Delay $>$ FIFO $\approx$ Capacity $\approx$ Fair $>$ fockey $>$ CSA. Delay delays the scheduling of a task in order to improve the data locality. Therefore, it violates more deadlines than all other schedulers. FIFO, Capacity and Fair schedule jobs without deadline awareness, but they do not delay scheduling a task when its data servers do not have computing slots. Therefore, they have better performance than Delay. Fockey and CSA schedule jobs with deadline awareness, so they have lower deadline violation rates than other schedulers. fockey only schedules submitted tasks, which may violate the deadlines of tight-deadline jobs when all computing slots are taken by loose-deadline tasks, as explained previously. The deadline-aware FIFO pre-scheduling in CSA avoids this kind of deadline violations. Also, we see that all schedulers except CSA have deadline violations when the number of jobs is no more than 24000 (i.e., the workload in [15]) in simulation and 800 in the real cluster. We also see that there are more deadline violations when there are more jobs and the increasing rate of CSA is slower than other schedulers. Therefore, $C S A$ has the best performance regarding deadline guarantee.

We measured the energy savings by the number of inactive servers. Our experimental results show that in all schedulers except CSA, there are no idle servers to be deactivated to save energy. This is because these schedulers use random data allocation first, which makes all servers store the requested data and either run tasks or respond to data requests. Therefore, we only show the number of inactive servers of CSA in simulation and the real cluster in Figures 7(a) and 7(b), respectively. We can see CSA achieves much energy savings. The energy savings decrease as the number of jobs increases because more jobs generate more workloads, which need more servers to run. The results indicate that CSA can save energy while other schedulers cannot when the cluster has extra computing capacity beyond the workload.

\subsection{Performance with Different Server Computing Capacities}

In this section, we varied the number of cores in a server from 4 to 9 with a step size of 1 in order to show the performance when servers have different computing capacities [10] Since a node in the real cluster has a fixed number of cores, we only measure the performance in simulation. In this experiment, each task requests one data block based on the default setting of Hadoop [31].

Figure 8(a) shows the network load of all schedulers. It shows the same order among all schedulers as in Figure 6(a) except that Delay generates the lowest network load. In Figure 6(a), each task requests 4 data blocks, and then with the random data allocation, all data blocks requested by a task are likely to be scattered among servers. Delay schedules a task to one of its data server that has the largest number of the task's requested data blocks, which generates a higher network load than CSA that allocates most request data blocks of a task to its data server due to job-first-data-second. In this experiment, since each task processes one data block, Delay can always schedule a task locally with its requested data by delaying its start time. Thus, Delay generates a lower network load than CSA. In order to provide deadline-guarantee, CSA cannot always put the tasks requesting the same data together. Some tasks run on servers other than their data servers in order to meet their deadlines at the cost of a certain network load. Figure 8(b) shows the deadline violation rate of all schedulers. It confirms that Delay generates a much larger deadline violation rate than CSA. Figures 8(b) and 8(a) indicate that CSA provides both high QoS and low network load compared to other schedulers.

We see that Figure 8(b) shows the same order among all schedulers as in Figure 6(a) due to the same reasons. We also can see that the deadline violation rate decreases as the computing capability of each server increases. This is because larger computing capability can handle more tasks at a time. The result indicates that CSA provides better QoS than all other schedulers when servers have different computing capacities.

\subsection{Effectiveness of Each Algorithm in $C S A$}

We measured the improved percentage of network load generated by CSA using cooperative data allocation (denoted by $N$ ) compared to the network load generated by CSA using random data allocation (denoted by $N^{\prime}$ ) [31] as $1-N / N^{\prime}$. Similarly, we measured the improved percentage of energy savings by $1-S^{a} / S^{a \prime}$. Figures 9(a) and 9 (b) show the average improved percentage of the energy savings and network load in simulation and the real cluster, respectively. We see that the cooperative data allocation greatly reduces the network load of the random data allocation because it allocates a data block to a server, which generates the lowest network load. The random data allocation leads to a much higher network load for data transmission. The figure also shows that the cooperative data allocation saves more energy than the random data allocation. This is because the cooperative data allocation algorithm allocates data requests to as few servers as possible to save energy. The results indicate that the cooperative data allocation is effective in increasing energy savings and reducing network load.

In order to measure the performance of recursive schedule refinement in improving data locality to achieve Objective (8), we changed the $\beta$ value from default 8 to 1 , which gives the same weight on improving data locality and energy savings. A lower $\beta$ value means the data locality should be improved at the cost of energy savings, which leads to more iterations in the recursive 


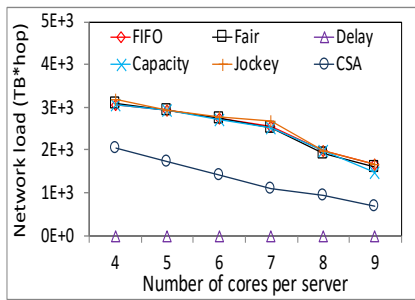

(a) Network load

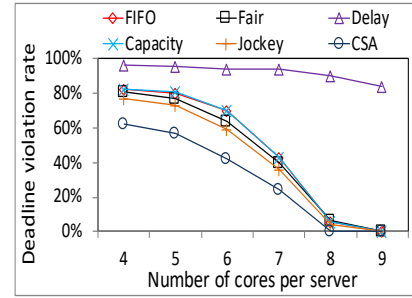

(b) Deadline violation

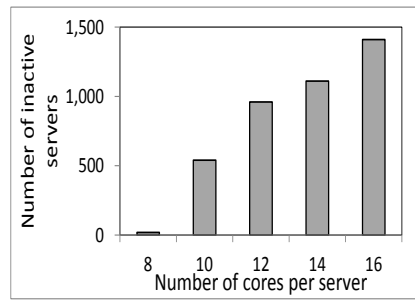

(c) Energy saving

Figure 8: Performance vs. server computing capability.

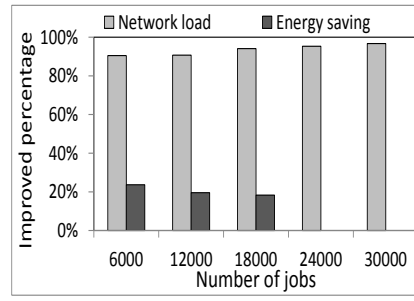

(a) Simulation

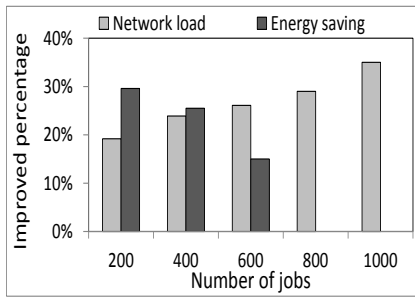

(b) Real cluster

Figure 9: Effectiveness of cooperative data allocation.

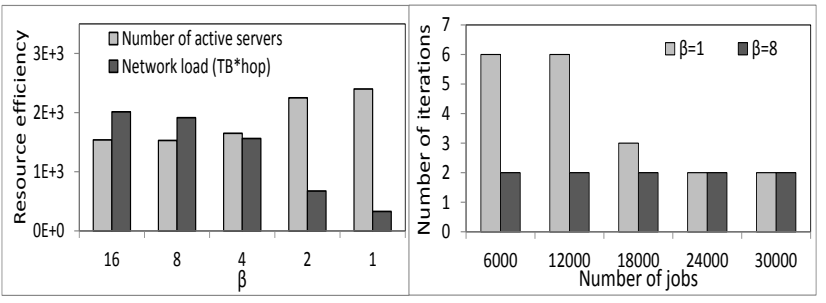

(a) Resource efficiency

(b) Number of iterations

schedule rigure 11: Effectiveness of $\beta$ in Formula (8). mized initially (as we deactivated all servers initially). Figures 10(a) and 10(b) show the network load and energy savings after each iteration for 6000 and 12000 jobs. The figures show that both network load and energy savings decrease over iterations during the refinement process. We see that more iterations lead to lower network load at the cost of energy savings since the task schedule refinement algorithm in the recursive schedule refinement process activates more inactive servers in order to improve the data locality. The results indicate that the recursive schedule refinement process is effective in achieving the tradeoff between data locality and energy savings in Objective (8).

Figure 11(a) shows the energy cost (measured by the number of active servers) and network load generated by $\operatorname{CSA}$, when $\beta$ is varied from 16 to 1 . It shows that the network load decreases and energy cost increases as $\beta$ decreases. A smaller $\beta$ gives more weight on network load in Objective (8). Each iteration reduces certain network load but increases the energy cost. Therefore, a smaller $\beta$ leads to lower network load, smaller energy savings and a larger number of iterations.

A larger number of iterations lead to more computing time of $C S A$. Figure 11(b) shows the number of iterations of CSA's recursive schedule refinement process versus different number of jobs in order to show the convergence speed of the refinement process to the final optimal results. We see that there are at most 6 iterations

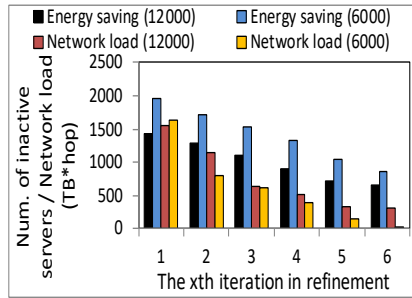

(a) Simulation

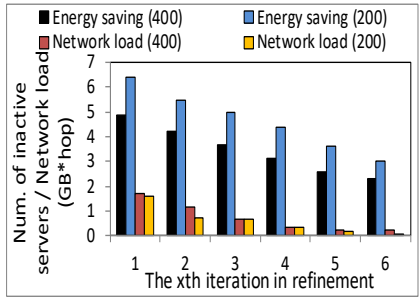

(b) Real cluster
Figure 10: Effectiveness of recursive schedule refinement process.

and a smaller $\beta$ leads to more iterations due to the same reasons as in Figure 11(a). It also shows that fewer jobs lead to a larger number of iterations when $\beta=1$. This is because more jobs use more active servers to support their computing workloads and then have fewer inactive servers to activate to improve data locality, leading to fewer iterations.

\subsection{Schedule Calculation Time}

Figure 12 shows the schedule calculation time of all schedulers versus the number of jobs. The scheduling time follows CSA>CSA_NR $>$ Capacity $\approx$ Fair $\approx C S A \_N D R>C S A \_$Online $>$fockey $\approx F I F O \approx$ Delay. $C S A \_N D R$ generates a comparable scheduling time to the Fair and Capacity schedulers, which means that CSA's job scheduler has similar scalability as Fair and Capacity. CSA_Online generates lower scheduling time than Fair and Capacity, which means it causes less influence on the job running latency. Fockey, FIFO and Delay generate lower scheduling time since they do not consider the fairness as Fair and Capacity or the task yielding as CSA_NDR. CSA_NR generates the second longest scheduling time because of the cooperative data allocation. CSA generates the longest scheduling time due to its extra recursive process. The figure indicates that the job scheduler in $C S A$ has similar scalability as other schedulers (when we considering $C S A$ 's offline scheduling). Though CSA has additional coop-

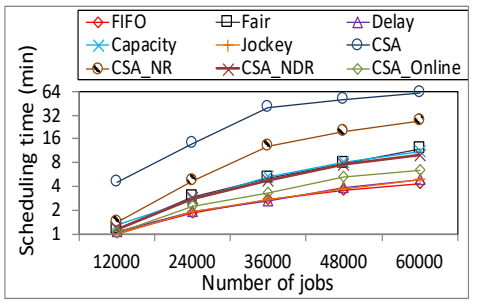

Figure 12: Schedule calculation time. erative data allocation and recursive schedule refinement, since all algorithm are offline scheduling, they do not introduce extra delay. 


\section{RELATED WORK}

Locality-aware job schedulers. Many job schedulers [1, 2, 31, 32, 43] try to schedule a task to its data server for data locality. The default Hadoop system [31] schedules jobs in the order of FIFO. The capacity scheduler [1] and the fair scheduler [2] ensure the fair sharing of computing resources among jobs and users. The coupling scheduler [32] also minimizes the data transmission during the shuffle stage to further improve data locality. The delay scheduler [43] delays scheduling of a task until one of its data servers has an available computing slot. Corral [25] places a job's tasks and their requested data into the same rack before the job's submission and places different jobs into different racks to expedite the process. Corral cannot improve data locality when the cluster is under heavy workload. Wang et al. [39] proposed a job queuing architecture and a map task scheduling algorithm to strike the right balance between data locality and load balancing. The above schedulers do not consider to provide deadline-guaranteed service.

Deadline-aware job schedulers. The deadline-aware schedulers focus on meeting the deadline requirements for jobs. Conductor [41] predicts the resource demands of MapReduce jobs, and automatically selects a computing service in clouds with monetary cost minimization to deploy the jobs to meet their deadlines. Gupta et al. [22] proposed a preemption algorithm that evicts jobs based on their deadlines. Jockey [19] profiles the execution time of jobs offline, and firstly schedules tasks of un-submitted jobs with tight-deadlines. Munir [27] leverages the job completion time prediction according to the network condition to minimize the average job completion time. Cheng et al. [16] proposed a resource and deadline-aware Hadoop job scheduler that estimates job completion times and predicts future resource availability to minimize job deadline misses. Chen et al. [14] proposed a MapReduce scheduler, which can obtain the optimal solution of the deadline-constrained scheduling problem by transforming the problem into a well-known graph problem. However, none of the above job schedulers cooperate with data allocation. Thus, they cannot achieve a resourceefficient schedule with maximized energy savings and data locality.

Data allocation. Current data allocation methods [9, 12, 31, 40] in computing clusters focus on data availability to handle machine failures. In Hadoop [31], three replicas are created for each data block, and each data replica is randomly assigned to a server Wang et al. [40] proposed a scalable block storage system, which uses replication techniques to improve data availability when writes fail. Amur et al. [9] proposed cost-effective method by turning off unnecessary servers to save power with data availability guarantee. Bonvin et al. [12] proposed a cost-efficient self-organized data replication method to ensure the data availability by adaptively adding new storage according to node failures. Different from these works, CSA has a cooperative data allocation algorithm, which is aware of computing load distribution over a cluster and conducts data allocation accordingly to improve resource utilization.

\section{CONCLUSION}

In this paper, we aim to meet the deadline requirement of users and the high resource-efficiency requirement of the cluster in a busy data-intensive parallel computing cluster. We propose CSA, a Cooperative job Scheduling and data Allocation method. CSA reverses the order of data allocation and job scheduling in current computing frameworks. Its requester-consolidation deadlineaware pre-scheduling algorithm improves job performance and energy savings. Based on the schedule of tasks, CSA's cooperative data allocation algorithm improves data locality, and its recursive schedule refinement algorithm further improves the performance and also achieves the tradeoff between energy savings and data locality with specified weights required by the cluster operator. The trace-driven experiments on both simulation and a real computing cluster show that CSA outperforms other schedulers in achieving deadline guarantee, data locality and energy savings simultaneously.

\section{ACKNOWLEDGEMENTS}

This research was supported in part by U.S. NSF grants NSF-1827674, CCF-1822965, OAC-1724845, CNS-1733596 and ACI-1661378, and Microsoft Research Faculty Fellowship 8300751.

\section{REFERENCES}

[1] [n. d.]. Apache Capacity Scheduler Guide. In http://hadoop.apache.org/docs/r1.2.1/capacity_scheduler.html [accessed in Apr. 2019].

[2] [n. d.]. Apache Fair Scheduler. In http://hadoop.apache.org/docs/r1.2.1/fair_ scheduler.html [accessed in Apr. 2019].

[3] [n. d.]. Apache Hadoop FileSystem and its Usage in Facebook. In http://cloud.berkeley.edu/data/hdfs.pdf [accessed in Apr. 2019].

[4] [n. d.]. Apache Hadoop NextGen MapReduce (YARN). In http://hadoop.apache.org/docs/r2.5.1/hadoop-yarn/hadoop-yarn-site/ [accessed in Apr. 2019].

[5] [n. d.]. Apache Spark. In https://spark.apache.org/. [accessed in Apr. 2019].

[6] [n. d.]. MESOS. In http://mesos.apache.org/ [accessed in Apr. 2019].

[7] [n. d.]. Palmetto Cluster. In http://citi.clemson.edu/palmetto/ [accessed in Apr. 2019].

[8] M. Al-Fares, A. Loukissas, and A. Vahdat. 2008. A Scalable, Commodity Data Center Network Architecture. In Proc. of SIGCOMM.

[9] H. Amur, J. Cipar, V. Gupta, and K. Schwan. 2010. Robust and Flexible PowerProportional Storage.. In Proc. of SoCC.

[10] R. Appuswamy, C. Gkantsidis, and A. Rowstron. 2013. Scale-up vs Scale-out for Hadoop: Time to rethink?. In Proc. of SoCC.

[11] A. Beloglazov and R. Buyya. 2011. Optimal Online Deterministic Algorithms and Adaptive Heuristics for Energy and Performance Efficient dDnamic Consolidation of Virtual Machines in Cloud Data Centers. (2011).

[12] N. Bonvin, T. G. Papaioannou, and K. Aberer. 2010. A Self-Organized, FaultTolerant and Scalable Replication Scheme for Cloud Storage.. In Proc. of SoCC.

[13] R. Chaiken, B. Jenkins, P. Larson, B. Ramsey, D. Shakib, S. Weaver, and J. Zhou. 2008. SCOPE: easy and efficient parallel processing of massive data sets. (2008).

[14] C. Chen, J. Lin, and S. Kuo. 2018. MapReduce scheduling for deadlineconstrained jobs in heterogeneous cloud computing systems. (2018).

[15] Y. Chen, A. Ganapathi, R. Griffith, and R. Katz. 2011. The Case for Evaluating MapReduce Performance Using Workload Suites.. In Proc. of MASCOTS.

[16] D. Cheng, J. Rao, C. Jiang, and X. Zhou. 2015. Resource and deadline-aware job scheduling in dynamic hadoop clusters. In Proc. of IPDPS.

[17] C. Delimitrou and C. Kozyrakis. 2015. Tarcil: reconciling scheduling speed and quality in large shared clusters. In Proc. of SOCC.

[18] M. Ehsan, Y. Chen, and H. Kang. 2013. EcoHadoop: A Cost-Efficient Data and Task Co-Scheduler for MapReduce. In Proc. of HiPC.

[19] A. D. Ferguson, P. Bodik, S. Kandula, E. Boutin, and R. Fonseca. 2013. Jockey: Guaranteed Job Latency in Data Parallel Clusters.. In Proc. of EuroSys.

[20] R. Gandhi, Y. C. Hu, C. Koh, H. H. Liu, and M. Zhang. 2015. Rubik: Unlocking the Power of Locality and End-point Flexibility in Cloud Scale Load Balancing.. In Proc. of USENIX ATC.

[21] M. R. Garey and D. S. Johnson. 1979. Computers and Intractability: A Guide to the Theory of NP-Completeness. W. H. Freeman.

[22] I. Gupta, B. Cho, M. R. Rahman, T. Chajed, N. Abad, C. L.and Roberts, and P. Lin. 2013. Natjam: Eviction Policies For Supporting Priorities and Deadlines in Mapreduce Clusters.. In Proc. of SoCC.

[23] B. Hindman, A. Konwinski, M. Zaharia, A. Ghodsi, A. D. Joseph, R. H. Katz, S. Shenker, and I. Stoica. 2011. Mesos: A Platform for Fine-Grained Resource Sharing in the Data Center.. In proc. of NSDI. 
[24] C. Hung, L. Golubchik, and M. Yu. 2015. Scheduling jobs across geo-distributed datacenters. In Proc. of SOCC.

[25] V. Jalaparti, P. Bodik, I. Menache, S. Rao, K. Makarychev, and M. Caesar. 2015 Network-Aware Scheduling for Data-Parallel Jobs: Plan When You Can. In Proc. of SIGCOMM.

[26] H. Li, A. Ghodsi, M. Zaharia, S. Shenker, and I. Stoica. 2014. Tachyon: Reliable memory speed storage for cluster computing frameworks. In Proc. of SOCC.

[27] A. Munir, T. He, R. Raghavendra, and F. Le. 2016. Network Scheduling Aware Task Placement in Datacenters. In Proc. of CONEXT.

[28] C. Peng and Z. Zhang. 2012. VDN: Virtual Machine Image Distribution Network for Cloud Data Centers.. In Proc. of INFOCOM.

[29] Q. Pu, G. Ananthanarayanan, P. Bodik, S. Kandula, A. Akella, P. Bahl, and I. Stoica. 2015. Low latency geo-distributed data analytics. (2015).

[30] S. Seny, J. R. Lorch, R. Hughes, C. G. J. Suarez, B. Zill, W. Cordeiroz, and J. Padhye. 2012. Don't Lose Sleep Over Availability: The GreenUp DecentralizedWakeup Service. In Proc. of NSDI.

[31] K. Shvachko, H. Kuang, S. Radia, and R. Chansler. 2010. The Hadoop Distributed File System. In Proc. of MSST.

[32] J. Tan, X. Meng, and L. Zhang. 2013. Coupling task progress for mapreduce resource-aware scheduling. In Proc. of INFOCOM.

[33] P. Vagata and K. Wilfong. [n. d.]. Scaling the Facebook data warehouse to 300 PB. In https://code.facebook.com/posts/229861827208629/scaling-thefacebook-data-warehouse-to-300-pb/.

[34] C. J. van Rijsbergen. 1979. Information Retrieval. Butterworth.

[35] V. Vavilapalli, A. C. Murthy, C. Douglas, S. Agarwal, M. Konar, R. Evans, T Graves, J. Lowe, H. Shah, and S. Seth. 2013. Apache hadoop yarn: Yet another resource negotiator. In Proc. of SOCC.

[36] H. Wang and H. Shen. 2018. Proactive Incast Congestion Control in a Datacenter Serving Web Applications. In Proc. of INFOCOM.

[37] H. Wang, H. Shen, and Z. Li. 2018. Approaches for Resilience Against Cascading Failures in Cloud Datacenters. In Proc. of ICDCS.

[38] H. Wang, H. Shen, and G. Liu. 2017. Swarm-based Incast Congestion Control in Datacenters Serving Web Applications. In Proc. of SPAA.

[39] W. Wang, K. Zhu, L. Ying, J. Tan, and L. Zhang. 2016. Maptask scheduling in mapreduce with data locality: Throughput and heavy-traffic optimality. (2016).

[40] Y. Wang, M. Kapritsos, Z. Ren, P. Mahajan, J. Kirubanandam, L. Alvisi, and M. Dahlin. 2013. Robustness in the Salus Scalable Block Store. In Proc. of NSDI.

[41] A. Wieder, P. Bhatotia, A. Post, and R. Rodrigues. 2012. Orchestrating the Deployment of Computations in the Cloud with Conductor.. In Proc. of NSDI.

[42] N. J. Yadwadkar, G. Ananthanarayanan, and R. Katz. 2014. Wrangler: Predictable and faster jobs using fewer resources. In Proc. of SOCC.

[43] M. Zaharia, D. Borthakur, J. Sen Sarma, K. Elmeleegy, S. Shenker, and I. Stoica 2010. Delay Scheduling: A Simple Technique for Achieving Locality and Fairness in Cluster Scheduling. In Proc. of EuroSys. 